\title{
Self-Learning Load Characteristic Models for Smart Appliances
}

\author{
Bruce Stephen, Member, IEEE, Stuart Galloway and Graeme Burt, Member, IEEE
}

\begin{abstract}
It is generally accepted that if dynamic electricity pricing tariffs were to be introduced, their effectiveness in controlling domestic loads will be curtailed if consumers were relied on to respond in their own interests. The complexities of relating behavior to load to price are so burdensome that at least some degree of automation would be required to take advantage of pricing signals. However, a major issue with home automation is fitting in with the lifestyles of individual consumers. Truly smart appliances that can learn the details of their routine operation may be several years away from widespread adoption making integrated home energy management systems unfeasible. Similarly, usage patterns of these same appliances may be substantially different from household to household. The contribution of this paper is the proposal and demonstration of a set of probabilistic models that act in a framework to reduce appliance usage data into contextual knowledge that accounts for variability in patterns in usage. Using sub-metered load data from various domestic wet appliances, the proposed technique is demonstrated learning the appliance operating likelihood surfaces from no prior knowledge ${ }^{1}$.
\end{abstract}

Index Terms - smart home, smart grid, demand response, energy management.

\section{INTRODUCTION}

As environmental awareness grows across the developed world, energy utility companies have been motivated by government legislation and public opinion to operate their infrastructure with greater efficiency in order to reduce generation requirements. A considerable source of inefficiency is the need for spinning reserves of generation to meet uncertain demand, in particular, overestimates to accommodate peak loads. It has been identified that a better understanding of loads would allow margins to be shrunk. Despite making up a significant portion of electricity demand [1-3] relatively little is known about domestic loads; the absence of metering on the low voltage circuit coupled with the traditional means of selling electricity, quarterly billing in the UK, means that there is very little being measured. A key technology in Smart Grids is the facility of Demand Response

\footnotetext{
1 This work was supported in part by the EPSRC Supergen Highly Distributed Energy Futures Programme (HiDEF).

B. Stephen is with the Institute for Energy \& Environment at Strathclyde University, Glasgow, U.K (bruce.stephen@strath.ac.uk).
}

(DR). The primary motivator for DR is system efficiency removing the need for significant generation margins in reserve to accommodate peaks in demand and thus preserve continuity in supply.

However, burdening the householder with the potentially complex task of deferring loads and checking pricing signals will not result in a robust and sustainable DR program. [4] deployed custom built clothes driers with demand response control circuitry built into to the appliance to allow signaled load shedding. This was possible with an appliance with such a simple duty cycle, but implementing true Smart Appliance intelligence should have the ultimate objective of operating domestic appliances at the optimal price at the most convenient time. If such appliances were to come to be commonplace, then they would need some kind of adaptive intelligence to cope with the dynamic nature of domestic energy use. This may comprise identifying usage times and learning the variability associated with them. Combining operating characteristics with household preference and tariff information could allow the deferring of appliance operation to a cost optimal time by automation.

Although ubiquitous Smart Appliances may be several years away, utility companies keen to avoid costly network reinforcements through the implementation of DR programs require these in timelier manner, a realization that may be possible through intermediary technology and retrofits. There have been two distinct approaches to monitoring the use of domestic appliances: building the telemetry into the appliance or the non-intrusive approach where the overall load profile is disaggregated into its constituent appliance loads. The latter approach was pioneered by [5] and has seen several progressively sophisticated algorithms proposed for extracting appliance loads [6-8]. These techniques are computationally expensive compared with just metering the individual appliance due to the large amounts of data required and intensive signal processing used. [9] demonstrated high resolution monitoring of white goods which was later followed by [10] extended with illustrations of practical examples and highlighting the need for analytics. The manufacturer centric monitoring of [10] has longer term objectives; a shorter term benefit of this capability is to inform a home automation system for enabling demand response and management. [11] cites [12] as an example of how informing users about their energy use aids their understanding in how to reduce it extraction of tasks requires metered plugs as [13] and [14] 
describe. Moving from the generic monitor of [11] to the specialized monitor of [10] requires additional analytics software to tailor required functionality from basic hardware. In order to do this, there needs to be a self learning capability on the part of the monitor.

This paper proposes a Machine Learning solution for online learning of appliance usage characteristics from no prior knowledge of their duty cycles or times of use; this allows it to be employed with a number of types of appliances. Section II reviews demand response technologies and the services they can provide to future electricity distribution networks. In Section III demand characteristics of domestic dwellings are discussed and in particular, how certain appliance types influence overall energy usage, Section IV describes the methodology for simulating an active smart appliance operation by gathering sub-metered data. The characteristics of some high energy use appliances are included to illustrate the challenging diversity of the problem. As potential smart appliances of the future are going to have to be competitively priced, computing power backing up their 'intelligence' is likely to be limited, hence simple online learning of operating behavior is proposed. The Kalman Filter realization of Bayesian Filtering, commonly seen in Robotics owing to its computational efficiency, is reviewed in Section V. Section VI proposes a model for learning the probability of time and extent of use of arbitrary appliances from metering data; the specializations to Kalman Filtering and the accommodation of multiple operating regimes are also detailed in Section VII. Case studies are shown for typical wet appliances in Section VIII. Conclusions outline the opportunities for hardware implementation, the benefits of additional model refinements and further applications.

\section{DEMAND RESPONSE}

There are a number of demand response mechanisms such as peak clipping, valley filling which may be facilitated by load shifting and longer term measures such as efficiency and electrification which stem from retrofit investment. Demand Response may be incentivized through time of use tariffs.

[15] estimated that in an urban DR trial in Norway, some $4.2 \%$ of demand peaks could be cut. [16] also noted the effectiveness of such schemes with a reduction in demand with $5 \%$ removed from peak demand. In [15] a DR study was conducted over a group of customers in Central Norway; this publication also noted that electricity was a 'low interest' product - one which the consumer had little comprehension as to the effect their habit driven lifestyles had on their level of usage. Alternatives to the flat rate averaged wholesale tariff as noted by [16, 17] include real time pricing, critical peak pricing and time of use pricing. Since retail price will be influenced by demand, available generation and generation costs, the flat rate cannot reflect the true cost of electricity at any point in time: peak will be subsidized by off-peak. Additionally, parsimonious users will not be rewarded for their frugality. A solution to this is a conservation rates model which thresholds consumption into blocks; such tariffs bring not only cost benefits to consumers but also, through reduced load, bring operational benefits to utilities by reducing load on plant at peak times. An additional aspect exists in the case of islanded power systems or those with constrained generation capacity e.g. [18]: alignment of supply and demand through the decision to store, export or consume energy from indigenous renewable generation is critical to security of supply.

\section{RESIDENTIAL DEMAND}

Domestic energy use accounted for around $34 \%$ of the total UK energy use in 2012 (Q2) [19], of this, typically 16-17\% can be attributed to wet appliances [1]. Figure 1 shows wet appliance usage in context with the overall energy use of a particular dwelling.

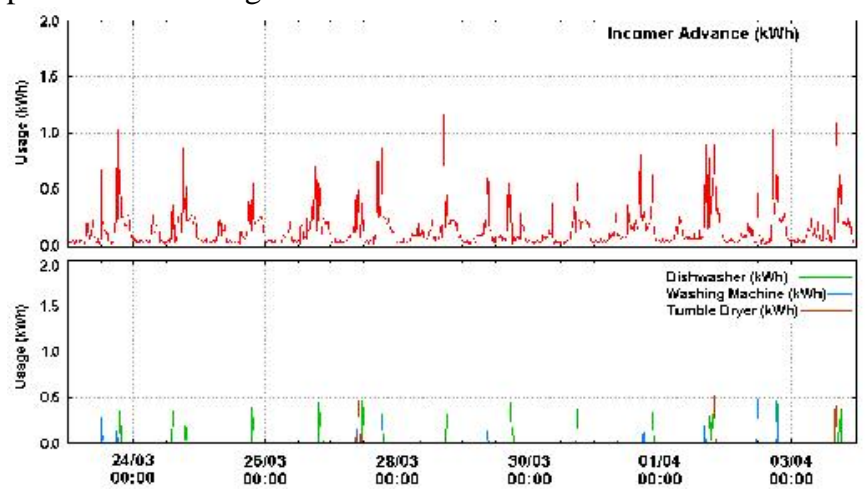

Fig. 1. Wet appliance operation against incomer.

Figure 1 shows a single dwelling metered at 30 minute intervals with the wet appliances, a dishwasher, washing machine and tumble dryer, sub-metered to indicate their contribution to the overall household load. These can be seen to contribute to up to $50 \%$ of a peak in load at times, offering significant potential for demand response participation. Although all in the same appliance category, all three appliances are used very differently and will most likely differ greatly again between dwellings [20] which highlights the need for monitoring over assumptions.

\section{Monitoring APPliAnCE EnERgy USAGE}

Rather than modify an existing appliance which may be technically infeasible, Smart Appliances are simulated through sub-metering of un-modified domestic wet appliances. The number of occupants in the property or their times of occupation were not recorded as this was intended capture appliance usage rather than the relationship between behavior routine and appliance usage. See [3] and [21] for detailed studies of dwelling utilization in this field.

\section{A. Metering Equipment}

Figure 2 shows the sub-metering arrangement which the appliances simply plug through which capture $\mathrm{kWh}$, peak $\mathrm{kW}$ and time of usage at 1 minute intervals. The overall consumption for the property was captured using a similar 
meter supplied with a current transducer clamp, which attaches to the tail of the meter for the property. This allowed the contributions of each appliance as well as the aggregated load to be tracked and if necessary disaggregated to explore hypothetical shifts in load. Sampling rates and data acquisition were controlled over a Zigbee Mesh network which allowed unobtrusive monitoring of appliances.

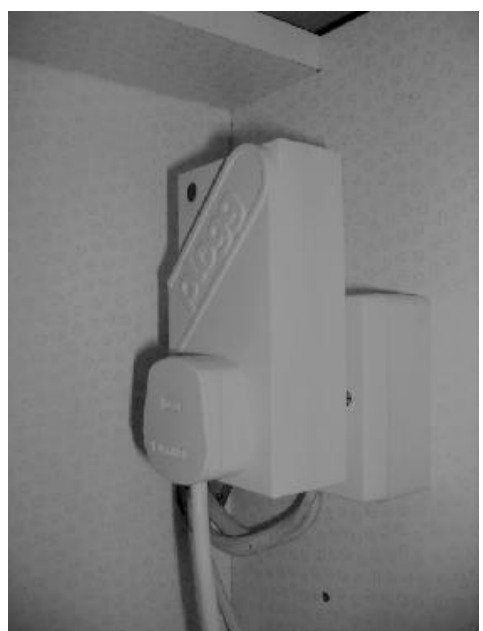

Fig. 2. Plug based appliance sub-meter in a UK domestic socket

Although 1-minute data was recorded for illustration, it is most likely that short term advantages would be gained from 15 or 30 minute advances which can be recovered by summation over measurements.

\section{B. Domestic Appliances Operation}

Standard domestic appliances were used in the trial; these were of various ages and were not necessarily new.

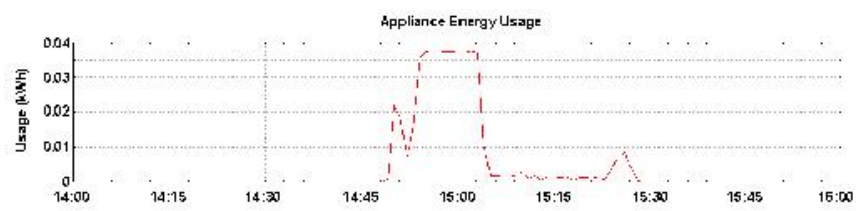

Fig. 3. Washing machine duty cycle sampled at 1 minute intervals.

Fig 3. Shows the duty cycle for a washing machine metered at 1 minute intervals to highlight the peaks in its energy usage. The usage of washing machines is driven by household need but this variability will be constrained by waking hours and work routines. It should be noted that this was not a new appliance and had several years of usage behind it which may have incurred additional variability in its load.

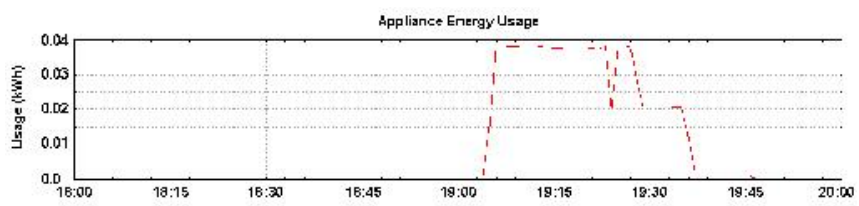

Fig. 4. Tumble dryer duty cycle sampled at 1 minute intervals.
Fig 4 shows a duty cycle of a Tumble Dryer. The Tumble Dryer is unusual in that it is driven by both routine and environmental factors (poor outdoor conditions) as well as being potentially coupled to other appliance operation (washing machine). The duty cycle is simpler, as it is limited only to heating the machines contents, which permits the usage to be interrupted, unlike washing machines and dishwashers whose more complex operations must be atomic to preserve correct operation. Also unlike washing machines and dishwashers, the duty cycle is of variable duration depending on size and composition of load. This further highlights the need to learn the behaviors that drive appliance energy usage. Figure 5 shows the duty cycles of a dishwasher; unlike the washing machine, this appliance is driven entirely by routine and one which is more consistent than laundry requirements.
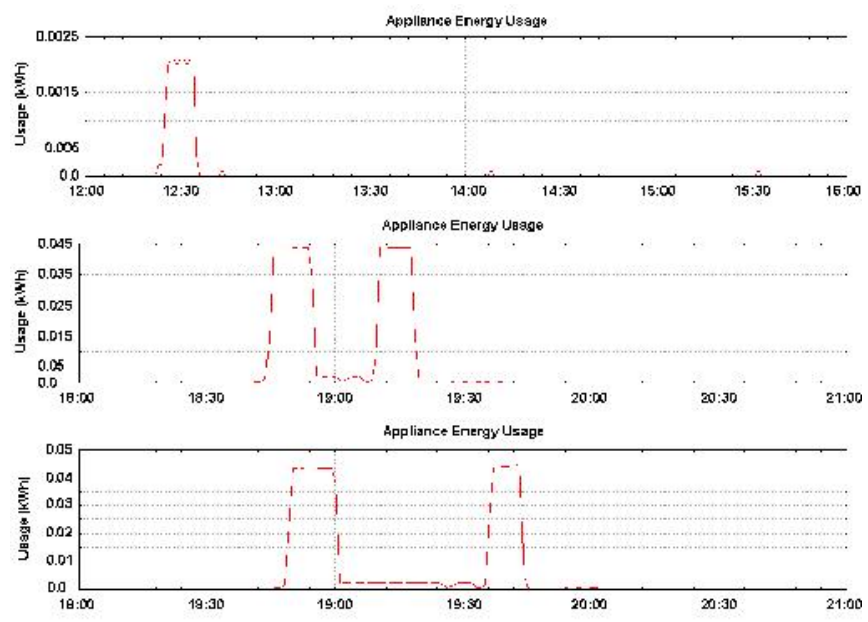

Fig. 5. Dishwasher duty cycles sampled at 1 minute intervals; from top: rinse, normal, intensive.

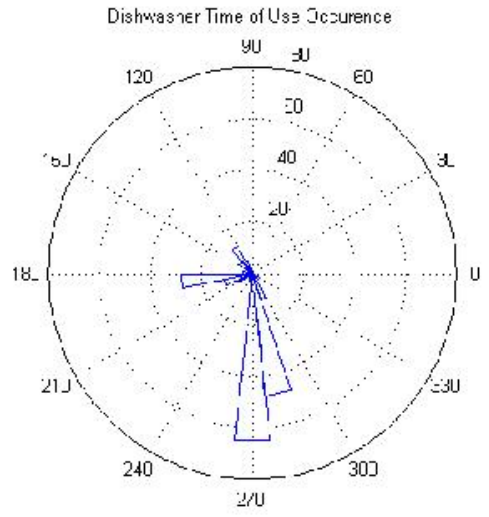

Fig. 6. Dishwasher usage time - 0 is midnight, 180 midday, and time advances counter clockwise.

Dishwashers are normally operated after mealtimes and as a result this will afford a greater degree of repeatability day on day. An alternative perspective is gained through looking at the times of operation over the course of a day for an appliance. Considering the same dishwasher over a one month period, Figure 6 shows a histogram of the times of usage and indicates a key part of the challenge of learning appliance operating characteristics, specifically that there are several 
behavioral regimes in operation apparent from the number of modes in the histogram: one which occurs at $6 \mathrm{pm}$ and is dominant and less frequent ones at $12 \mathrm{pm}$ and $8 \mathrm{am}$. A similar multi-modality is exhibited in the energy usage advance magnitude as shown in figure 7.

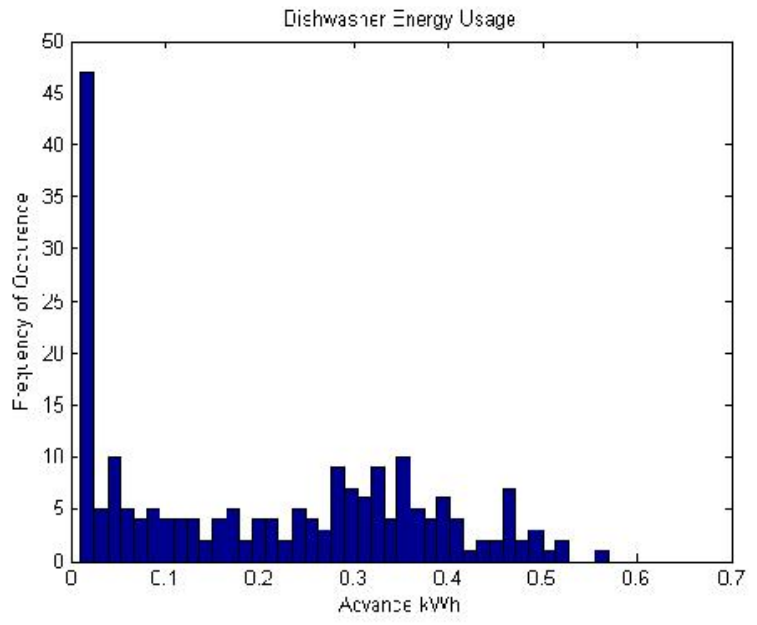

Fig. 7. Histogram of energy usage advances for a dishwasher.

The majority of 15 minute advances exhibit very small energy usages but there are also significant occurrences of higher levels of energy usage representing longer or more intense cycles.

\section{BAYESIAN FILTERING}

If an offline learning approach were taken to this problem, data storage would be required to archive exemplar data, the amount of exemplar data required would have to be known in advance and the form of the model would have to be specified; as noted in the previous two sections, this model is likely to incorporate multiple behavior regimes. Significant a-priori knowledge is therefore a prerequisite of offline learning, making it unfeasible: for a home automation controller to know, 'out of the box', how every appliance in a particular household works is nearly impossible given the variation in ratings and operating regimes that are possible within a single appliance type alone. Further variability is added through the inherent uncertainty in human behavior. For this reason the representation of the appliance operation must be learned and given the anticipated hardware and operational constraints must be learned online.

\section{A. Bayesian Filtering}

In recent years it has become more common to see uncertainty in systems being modeled using a Bayesian approach. This encodes all beliefs and uncertainties surrounding a process as probability distributions. One strategy to implement online learning of a systems state is to use Bayesian Filtering. The amounts to a recursive estimation of the posterior distribution of the mean with respect to past measurements [22]:
$P\left(z_{t} \mid x_{t}\right)=\int P\left(z_{t} \mid z_{t-1}\right) P\left(z_{t-1} \mid x_{t-1}\right) d x_{t-1}$

For a measurement $x$ and state $z$. The integral is intractable for most distribution forms and usually has to be approximated numerically. However, an analytical solution can be obtained for a Gaussian distribution which can be shown to have a formulation equivalent to the Kalman Filter [23]. The Kalman Filter amounts to an auto-adaptive estimator of a Gaussian posterior, the parameters of which correspond to the state variable $z$ that is essentially a mean $\mu$. The dynamics of the underlying process are assumed to be a Markov-Gauss state model i.e. current state is linearly related, through matrix $A$, to past state:

$z_{t}=A_{t} z_{t-1}+\varepsilon_{t}$

Added Gaussian process noise is drawn from:

$\varepsilon_{t} \sim N\left(0, R_{t}\right)$

Where $R$ is the process noise covariance; this leads to the following distribution being estimated:

$P\left(z_{t} \mid z_{t-1}\right)=N\left(z_{t} ; A_{t} z_{t-1}, R_{t}\right)$

Observations are linearly related to state through matrix $C$ as follows:

$x_{t}=C_{t} z_{t}+\delta_{t}$

As with the process model, Gaussian observation noise is given by:

$\delta_{t} \sim N\left(0, Q_{t}\right)$

Noise covariance is given by $Q$. This leads to the following distribution of observation likelihood:

$$
P\left(x_{t} \mid z_{t}\right)=N\left(x_{t} ; C_{t} z_{t}, Q_{t}\right)
$$

Assuming a first order Markov model and a fixed evolution, the recursive estimates of the Gaussian mean $\mu$ and covariance $\sum$ are as follows:

$$
\begin{aligned}
& \bar{\mu}_{t}=A_{t} \mu_{t-1} \\
& \bar{\Sigma}_{t}=A_{t} \Sigma_{t} A_{t}^{T}+R_{t}
\end{aligned}
$$

Assuming simple temporal dynamics allows matrices $A$ and $C$ to be fixed as identity matrices although these may also be 
learned from observation data. An update or corrective step is then performed as a new observation comes in:

$$
\begin{aligned}
& K_{t}=\bar{\Sigma}_{t} C_{t}^{T}\left(C_{t} \bar{\Sigma}_{t} C_{t}^{T}+Q_{t}\right)^{-1} \\
& \mu_{t}=\bar{\mu}_{t}+K_{t}\left(x_{t}-C_{t} \bar{\mu}_{t}\right) \\
& \Sigma_{t}=\left(I-K_{t} C_{t}\right) \bar{\Sigma}_{t}
\end{aligned}
$$

One problem with this model is that it assumes a single state (mean) whereas the operation of appliances may have multiple operating regimes that need to be accommodated.

\section{Probabilistic Modeling of Appliance Usage}

The ultimate need for such models will be to propose particular domestic demand estimates with their associated measure of certainty, which implies a probabilistic modeling approach. The predicates of such approaches are probability distributions representing the variables involved in the overall load. Bayesian modeling of probabilism differs from its more conventional Frequentist counterpart in its modeling of all variables, including distribution parameters through probability distributions. Although the basic form of the data implies the probability distribution choice, several factors can complicate this. Data that is said to be drawn from different probability distributions, albeit of the same form, is said to be non-stationary. Non-stationarity can result in multiple modes, skewing or multi-tangentiality all potentially stemming from distinct operating regimes. Given that human behavior is highly dynamic, the form of these cannot possibly be known apriori thus necessitating a self learning system that captures a representation of appliance operating characteristics over time. The following section highlights the aspects of operation that could be utilized.

\section{A. Usage Time}

Time, like direction, cannot be treated as other random variables as it 'wraps around' or is periodic [24]. Adopting the same assumptions for periodic space as real space leads to confounding results [24]. Employing this allows usage time hour $H$ and minute $m$, to be modeled by converting the time of day into a usage time of day angle $u$ :

$u=2 \pi \frac{60 H+m}{1440}$

The equivalent of the Gaussian distribution in periodic space is the von Mises distribution:

$$
P(u ; \omega, \kappa)=\frac{1}{2 \pi I_{0}(\kappa)} \exp \{\kappa \cos (u-\omega)\}
$$

The von Mises distribution is parameterized by centre $\omega$ and dispersal $\kappa$, equivalent to mean and variance respectively, in a linear space. $\mathrm{I}_{0}$ is the zeroth order Bessel function.

\section{B. Duration of Use}

Duration of use or advance size are strictly positive real numbers allowing a log normal distribution over load advance $l$, to be assumed:

$$
P\left(l ; \mu, \sigma^{2}\right)=\frac{1}{l} \frac{1}{\sqrt{2 \pi \sigma^{2}}} \exp \left\{\frac{(\ln l-\mu)^{2}}{2 \sigma^{2}}\right\}
$$

This is parameterized with mean $\mu$ and variance $\sigma^{2}$. These advances are over 15 minutes for the sake of demonstrating times of use in higher granularity - in practice the less demanding 30 minute advance would be used.

\section{Learning Wet APPliance OPERATING CHARACTERISTICS}

\section{A. Multiple Hypothesis Tracking}

The sequential estimate of a non-stationary mean does not permit the existence of multiple modes of operation, new conflicting operating regime characteristics will end up being smoothed into unrepresentative states if the basic linear Gaussian model is adopted. The solution here is to maintain multiple hypotheses simultaneously. If alternating occurrences of a particular regime were encountered, this would result in an operating state being estimated that fell between both regimes and represented neither one adequately. What would be required is the means of estimating not a single posterior distribution but multiple ones. Multiple Hypothesis Tracking (MHT) [25] techniques exist to accommodate situations such as this in radar/target tracking applications. Since the number of regimes cannot be known in advance, there needs to be a means of branching the original state estimates into a second hypothesis and another of selecting the hypothesis an observed measurement came from and updating it accordingly. Much of this functionality can be obtained by maintaining multiple Kalman Filters and assuming the posterior distribution can be approximated by a mixture of $G$ Gaussians:.

$$
P\left(x_{t}\right)=\sum_{g=1}^{G} \eta_{g} P\left(z_{t}\right)
$$

Where $\eta_{g}$ is the count of the number of times hypothesis $g$ has been seen. In systems where there are limited computational resources it may be necessary to lower bound this to excise infrequent hypotheses from the model. Commonly seen in agglomerative type clustering methods, the Salmond Distance $D$ [26] allows similar hypotheses to be merged:

$$
D(i, j)=\frac{\eta_{i} \eta_{j}}{\eta_{i}+\eta_{j}}\left(\mu_{i}-\mu_{j}\right)^{T} \Sigma_{i}^{-1}\left(\mu_{i}-\mu_{j}\right)
$$

Statistical testing of the Salmond Distance indicates when a new hypothesis occurs or rather the existing one is rejected.

\section{B. Domain Transforms}

For the appliance models, the state distributions are nonGaussian and cannot be estimated online. This shortcoming can be overcome by maintaining observation data in its Gaussian/Euclidean space form but then transforming the 
estimated parameters into their respective distribution spaces. The von Mises distribution can be approximated from the parameters of the Circular Normal thus allowing usage time to be captured recursively. In [27] it was shown that the recursive estimates of a Gaussian posterior could be used to estimate the directional mean $\omega$ and dispersion parameters $\kappa$ of a von Mises distribution at timestep $t$ :

$$
\begin{aligned}
& \omega_{t}=\arctan \left(\frac{s_{t}}{c_{t}}\right) \\
& \kappa_{t}=\sqrt{C_{t}^{2}+S_{t}^{2}} \\
& C_{t}=\Sigma_{t-1} \cos \mu_{t-1}+R_{t} \cos x_{t} \\
& S_{t}=\Sigma_{t-1} \sin \mu_{t-1}+R_{t} \sin x_{t}
\end{aligned}
$$

Where $R$ is the observation noise covariance. Similarly, for power usage $l$ at a particular time, the distribution is nonGaussian as a strictly positive constraint is observed. The updates to these Log Normal distribution parameters, mean $\mu$ and variance $\sigma^{2}$, can also be obtained by transforming the Kalman Filter state estimates as follows:

$$
\begin{aligned}
\frac{\mu_{t}}{\sigma_{t}^{2}} & =\frac{\mu_{t-1}}{\sigma_{t-1}^{2}}+\frac{\ln l_{t}}{R_{t}} \\
\frac{1}{\sigma_{t}^{2}} & =\frac{1}{\sigma_{t-1}^{2}}+\frac{1}{R_{t}}
\end{aligned}
$$

The combination of the transformed feature space, the state estimation and the hypothesis generation/pruning allows the time/energy likelihood surface to be tracked as it evolves.

\section{SMART APPLIANCES For DEMAND RESPONSE}

Time of use tariffs typically price energy at an hourly or half hourly intervals to align with the capabilities of domestic metering equipment and the operation of market balancing mechanisms [28]. Any interval could be used with the models presented here, but for the purposes of illustrating the ability to learn times of use to a greater resolution, 15 minute data is employed. Using a three dimensional observation Kalman Filter in the transformed space (20-23), the operating regimes of the previously discussed wet appliances can be learned for a given household. Given its strongly routine driven nature, the dishwasher is considered in detail here. Running the profile learning algorithm over online appliance data results in a collection of von Mises/Log Normal distribution pairs which can be aggregated linearly into a joint distribution over load magnitude and time of usage combinations. Figure 8 shows the von Mises distributions captured for usage time for the dishwasher.

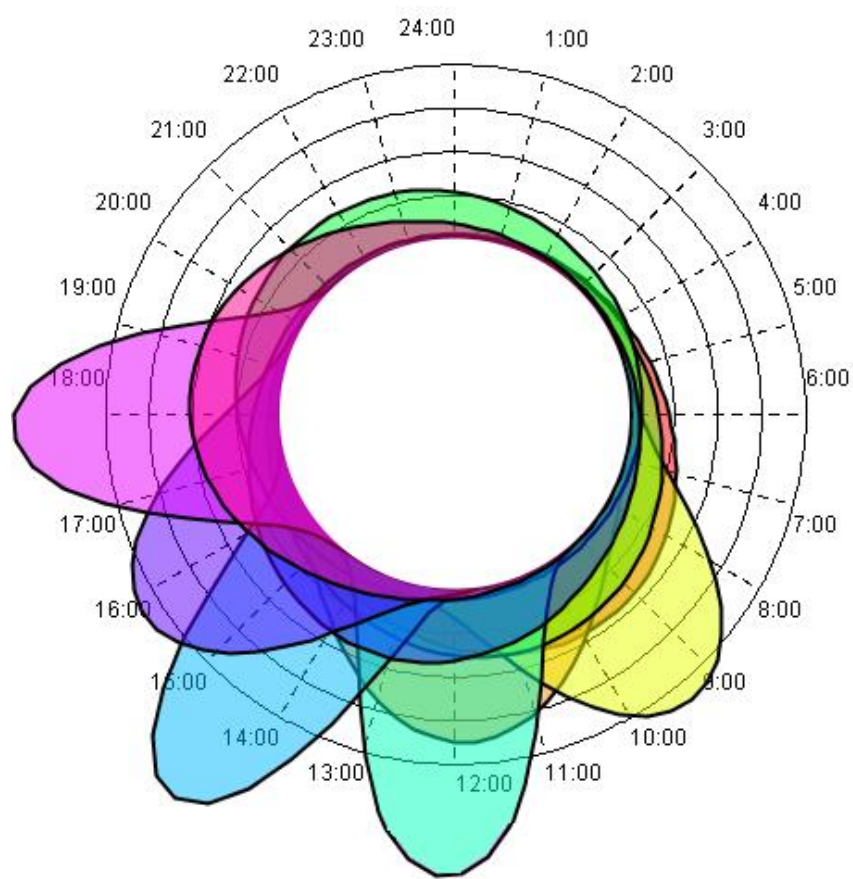

Fig. 8. Usage time probability distribution of dishwasher operation, learned over 1 month of operation - notable for its multi-modality.

The distributions are associated with distinct behavior regimes that correspond to household routine such as the operation of a rinse cycles in the morning and mid-day and a full wash cycle in the evening, The Log-Normal distributions of load associated with these usage times are shown in figure 9.

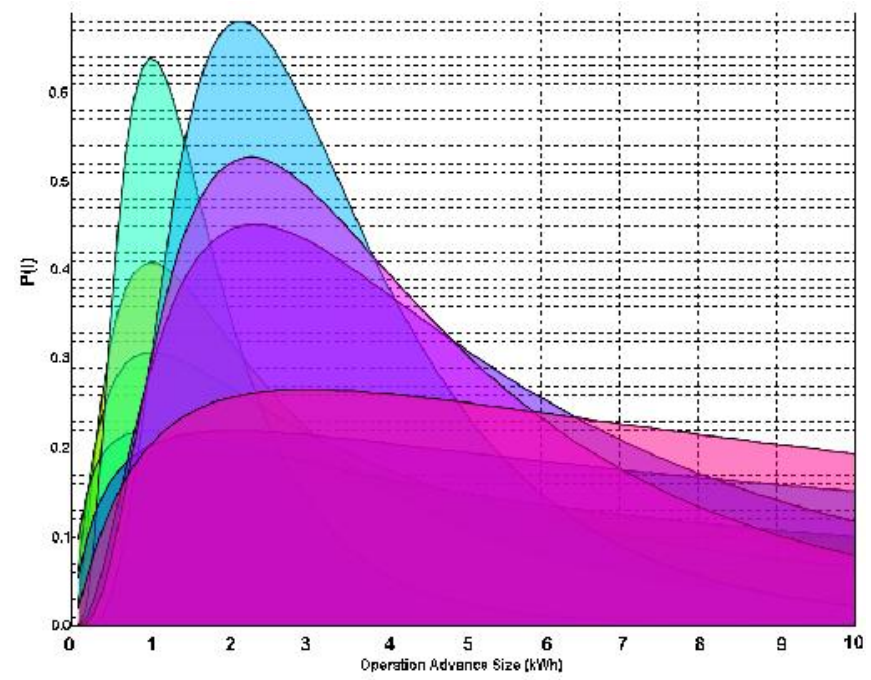

Fig. 9. Energy use probability distribution of dishwasher, learned over 1 month of operation.

Taking the product of the distributions shown in Figures 8 and 9 results in a joint density like the one represented with a heat map in figure 10 which indicates the joint probability of load magnitude $l$ and usage time $u$ obtained from

$P(u, l)=\sum_{g=1}^{G} \eta_{g} P\left(u ; \omega_{g}, \kappa_{g}\right) P\left(l ; \mu_{g}, \sigma_{g}^{2}\right)$

No dependency structure is assumed between $u$ and $l$. 

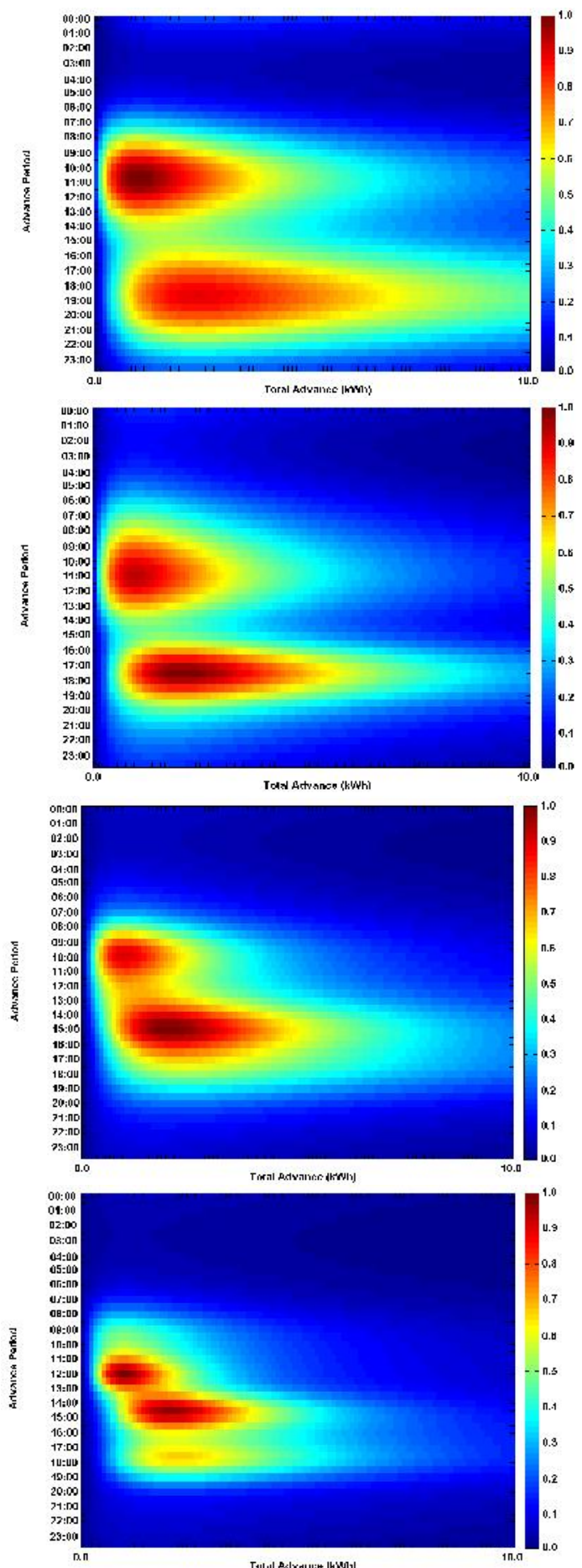

Fig. 10. Joint likelihood surface of dishwasher operation learned after (from top), 1, 7, 28 and 31 days (complete monitoring period) operation. Color intensity represents increasing joint probability of observing a particular level of usage at a particular time.

Figure 8 shows the regions of high probability density for usage time - as expected these form several peaks or modes. Figure 10 demonstrates how the learned likelihood surface for a dishwasher refines progressively over a number of days, adjusting as the observed use of the appliance changes. What this distribution will do for a Smart Home/Smart Grid is provide the likely usage time/load magnitude combinations for a DR strategy to operate on - loads cannot be shed if they do not exist, may not provide a useful demand service if operated for too short a time, or may be unsuited to providing any kind of service reliably given the high variability of their operation. Even at the early stages, a clear set of behaviors emerge, manifesting themselves as high likelihood regions, reflecting lunch, dinner and breakfast invoked operating regimes as shown in figure 8 . As more observations are added over time, the usage model becomes progressively less ambiguous; the observation likelihood of the Kalman Filter, given by (7), measures this ambiguity explicitly.

TABLE I

SAMple Model PARAmeters Learned From DishWASher OPERATION

Operational regimes learned from 228 operating cycles of a domestic dishwasher.

\begin{tabular}{|c|c|}
\hline Mean Usage time (HH:mm) & Mean Total Advance Size \\
\hline 9:19 & $0.02 \mathrm{kWh}$ \\
\hline $9: 41$ & $0.02 \mathrm{kWh}$ \\
\hline $10: 40$ & $0.021 \mathrm{kWh}$ \\
\hline 11:50 & $0.02 \mathbf{k W h}$ \\
\hline $12: 40$ & $0.02 \mathrm{kWh}$ \\
\hline $13: 12$ & $0.735 \mathrm{kWh}$ \\
\hline $14: 26$ & $0.768 \mathrm{kWh}$ \\
\hline $15: 48$ & $0.852 \mathrm{kWh}$ \\
\hline $17: 52$ & $0.827 \mathrm{kWh}$ \\
\hline $18: 20$ & $1.11 \mathrm{kWh}$ \\
\hline 21:18 & $0.02 \mathrm{kWh}$ \\
\hline
\end{tabular}

Table I shows these 11 operating regimes recovered by the Kalman Filter as mixture components (i.e. $G=11$ in (16)) from the single month of usage data. As can be seen from usage times, distinct patterns have emerged here that can be attributed to the domestic routine of the householder.

\section{CONCLUSION}

This paper has proposed and demonstrated models that learn probabilistic representations of appliance operation and use for inclusion in future 'Smart' products that can aid and automate domestic demand response. These models would be useful in constrained grid situations such as rural feeders or islanded power systems. Anticipating demand levels with an associated confidence could allow a substation computer to bring on or off loads such as EV chargers or wet appliances within the convenience bounds of the consumers routine. Provision of demand response at higher time resolutions, for example for frequency response, would require more detailed representations of appliance duty cycle. To achieve this, the complexities of dishwasher and washing machine load requirements over the course of their operation could be captured using a distribution approximated using a Hidden Markov Chain [29] which could capture both the expected loads and the order in which these occurred. Aside from this, additionally accurate expectations could be achieved through consideration of more complex dependency structure than the 
independence currently assumed. Independence means all possible learned load expectations are possible at all expected times of use - which could be constrained by relaxing the independence assumption in (24). Copula statistics, usually applied to probabilistic models in finance have shown themselves to be applicable in other domains where non-linear dependency relations are required [30, 31] - these work through abstracting the dependency model away from the marginal probabilities of the individual variables in a model; Dynamic Bayesian Networks [29] also offer an adaptive solution to learning this relation possibly through piecewise approximation of complex dependency.

\section{REFERENCES}

[1] Hamidi, V., Li, F. \& Robinson, F., "Demand response in the UK's domestic sector", Electric Power Systems Research 79, pp. 1722-1726. Elsevier, 2009.

[2] Yao, R. \& Steemers, K., "A method of formulating energy load profile for domestic buildings in the UK". Energy and Buildings 37, pp. 663 671. Elsevier 2005

[3] Roscoe, A.J. \& Ault, G. "Supporting high penetrations of renewable generation via implementation of real-time electricity pricing and demand response". IET Renewable Power Generation, Vol. 4, Issue. 4, pp. 369-382, 2010.

[4] Hammerstrom, D.J. et al. Pacific Northwest Gridwise Test bed demonstration projects part 2: Grid Friendly Appliance Project. PNNL Report no.17079, Pacific Northwest National Laboratory, Richland, Washington; October 2007.

[5] Hart, G.W., "Non-intrusive Appliance Load Monitoring". Proceedings of the IEEE, Vol. 80, No. 12, December 1992.

[6] Zeifman, M. \& Roth, K. "Nonintrusive Appliance Load Monitoring: Review and Outlook", IEEE Transactions on Consumer Electronics, Vol. 57, No. 1, pp. 76-84, February 2011.

[7] Liang, J., Ng, S.K.K., Kendall, G. \& Cheng, J.W.M., "Load Signature Study_Part I: Basic Concept, Structure, and Methodology". IEEE Transactions on Power Delivery, Vol. 25, No. 2, April 2010.

[8] Liang, J., Ng, S.K.K., Kendall, G. \& Cheng, J.W.M., "Load Signature Study-Part II: Disaggregation Framework, Simulation, and Applications". IEEE Transactions on Power Delivery, Vol. 25, No. 2, April 2010.

[9] Simon, M., Bee, G., Moore, P., Pu, J. \& Xie, C. "Modelling of the life cycle of products with data acquisition features". Computers in Industry 45, pp. 111-122, Elsevier 2001.

[10] Yang, X., Moore, P., Wong, C. \& Pu, J. “A Component-based Software Framework for Product Lifecycle Information Management for Consumer Products". IEEE Transactions on Consumer Electronics, Vol. 53, No. 3, August 2007.

[11] Cho, H.S., Yamazaki, T. \& Hahn, M. "AERO: Extraction of Users Activities from Electric Power Consumption Data”, IEEE Transactions on Consumer Electronics, Vol. 56, No. 3, August 2010.

[12] Darby, S., "The effectiveness of feedback on energy consumption: A review for DEFRA of the literature on metering, billing and direct displays", Environmental Change Institute, University of Oxford, 2006.

[13] Cho, H.S., Yamazaki, T. \& Hahn, M., "Determining Location of Appliances from Multi-hop Tree Structures of Power Strip Type Smart Meters". IEEE Transactions on Consumer Electronics, Vol. 55, No. 4 November 2009.

[14] Cho, H.S., Yamazaki, T. \& Hahn, M., "Simple and Robust method for Location Detection of Electric Appliances using Markers and Programmable Logic Devices”. Proceedings of ICSE 2009, pp. 334-338, 2009.

[15] Saelle, H. \& Grande, O.S., "Demand Response from Household Customers: Experiences from a Pilot Survey in Norway". IEEE Transactions on Smart Grid, Vol. 2, No. 1, March 2011.

[16] Hammerstrom, D.J. et al. Pacific Northwest Gridwise Test bed demonstration projects part 1: Olympic Peninsula Project. October 2007.
[17] Mohsenian-Rad, A. \& Leon-Garcia, A., "Optimal Residential Load Control With Price Prediction in Real-Time Electricity Pricing Environments", IEEE Transactions on Smart Grid, Vol. 1, No. 2, September 2010

[18] Gill, S., Dolan, M., Frame, D. \& Ault, G. (2011) The role of the electric heating and district heating networks in the integration of wind energy to Island networks. International Journal of Distributed Energy Resources, 7 (3). 245 - 263. ISSN 1614-7138

[19] DECC, UK Energy in Brief 2012 available at: http://www.decc.gov.uk/assets/decc/11/stats/publications/energy-inbrief/5942-uk-energy-in-brief-2012.pdf

[20] Gram-Hanssen, K., "Standby consumption in households analysed with a practice theory approach", Journal of Industrial Ecology, Vol. 14, No. $1,2009$.

[21] Richardson, I., Thomson, M., Infield, D. \& Clifford, C. "Domestic electricity use: A high-resolution energy demand model”, Energy and Buildings 42, pp. 1878-1887, Elsevier 2010.

[22] Chen, Z., "Bayesian filtering: From Kalman filters to particle filters, and beyond." Statistics (2003): 1-69.

[23] Kalman, R.E., "A New Approach to Linear Filtering and Prediction Problems", Transactions of the ASME--Journal of Basic Engineering, Volume 82, Series D, pp. 35-45, 1960.

[24] Mardia, K.V. \& Jupp, P.E. (2000) Directional Statistics, Wiley, Chichester.

[25] Blackman, S., "Multiple hypothesis tracking for multiple target tracking”, IEEE Trans. Aerosp. Electron. Syst. Mag. Tutor, Vol. 19, No. 1, pp. 5-18, 2004.

[26] Salmond, D.J., "Mixture Reduction Algorithms for Uncertain Tracking", Royal Aerospace Establishment, Farnborough, U.K., Tech. Rep.88004, Jan. 1988.

[27] Azmani, M., Reboul, S., Choquel, J.B. \& Benjelloun, M., "A recursive change point estimate of the wind speed and direction," Computational Cybernetics, 2009. ICCC 2009. IEEE International Conference on , vol., no., pp.51-56, 26-29 Jan. 2009

[28] Wilson, I.A., McGregor, P. G., Infield, D.G. \& Hall, P. J., "Gridconnected renewables, storage and the UK electricity market", Renewable Energy, Vol. 36, No. 8, pp. 2166-2170. Elsevier 2011.

[29] Ghahramani, Z., "An introduction to hidden Markov models and Bayesian networks." International Journal of Pattern Recognition and Artificial Intelligence Vol. 15 No.1, pp. 9-42, 2001.

[30] Stephen, B., Galloway, S.J., McMillan, D., Hill, D. C. \& Infield, D. G., "A copula model of wind turbine performance". IEEE Transactions on Power Systems, Vol. 26, No. 2, pp. 965-966. 2011

[31] Berkes, P., Wood, F. \& Pillow, J., "Characterizing neural dependencieswith copula models", Adv. Neural Inf. Process. Syst., Vol. 21, pp.129-136, 2009

\section{BIOGRAPHIES}

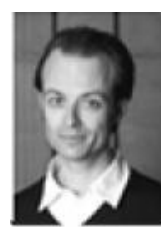

Bruce Stephen (M'09) currently holds the post of Senior Research Fellow within the Institute for Energy and Environment at the University of Strathclyde. He received his B.Sc. from Glasgow University and M.Sc. and $\mathrm{PhD}$ degrees from the University of Strathclyde. His research interests include Distributed Information Systems, Machine Learning applications in Power System and Agricultural Condition Monitoring and Asset Management as well as understanding energy end use.

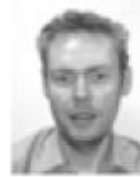

Stuart Galloway is a Senior Lecturer within the Institute for Energy and Environment. He obtained his MSc and $\mathrm{PhD}$ degrees in mathematics from the University of Edinburgh in 1994 and 1998 respectively. His research interests include power system optimization, numerical methods and simulation of novel electrical architectures.

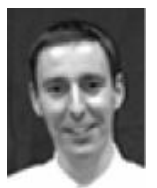

Graeme Burt (M'95) received his BEng in Electrical and Electronic Engineering and his $\mathrm{PhD}$ from the University of Strathclyde in 1988 and 1992 respectively. His research interests lie predominantly in the areas of power system modeling and simulation, power system protection, and network integration of distributed generation. 